\title{
Human Lung Cancer-Derived Immunosuppressive Plasmacytoid Dendritic Cells Release IL-1 $\alpha$ in an AIM2 Inflammasome-Dependent Manner
}

\author{
Rosalinda Sorrentino, ${ }^{*}$ Michela Terlizzi, ${ }^{*}$ Vincenzo G. Di Crescenzo, ${ }^{\dagger}$ Ada Popolo, ${ }^{*}$ Michela Pecoraro, ${ }^{*}$ Giuseppe Perillo, \\ Antonio Galderisi, ${ }^{\S}$ and Aldo Pinto* \\ From the Departments of Pharmacy* and Medicine and Surgery, ${ }^{\dagger}$ University of Salerno, Fisciano; and the Divisions of the Respiratory System, ${ }^{\ddagger}$ and \\ Pneumological and Bronchial Endoscopy, ${ }^{\S}$ A.O.U. San Giovanni di Dio e Ruggi D’Aragona, Salerno, Italy
}

\author{
Accepted for publication \\ July 9, 2015. \\ Address correspondence to \\ Rosalinda Sorrentino, Ph.D., \\ Department of Pharmacy, \\ University of Salerno, Via \\ Giovanni Paolo II 132, \\ Fisciano, 84084 Salerno, \\ Italy. E-mail: rsorrentino@ \\ unisa.it.
}

\begin{abstract}
Plasmacytoid dendritic cells ( $\mathrm{pDCs}$ ) highly populate lung tumor masses and are strictly correlated to bad prognosis, yet their role in lung cancer is controversial. To understand their role in lung cancer, we isolated pDCs from human samples of lung obtained from non-small cell lung cancer patients undergoing thoracic surgery. Tumor masses presented a higher percentage of pDCs than healthy tissues; pDCs were in the immunosuppressive phenotype, as determined by higher levels of CD33 and PD-L1. Despite higher HLA-A and HLA-D expression, cancerous PDCs did not exert cytotoxic activity against tumor cells but instead promoted their proliferation. In this scenario, cancerous pDCs were able to produce high levels of IL-1 $\alpha$. This effect was observed on the specific activation of the inflammasome absent in melanoma 2 (AIM2), which led to higher cytoplasmic calcium release responsible for calpain activation underlying IL-1 $\alpha$ release. The blockade of type I interferon receptor and of AIM2 via the addition of LL37 significantly reduced the release of IL-1 $\alpha$, which was still high after Nod-like receptor P3 inhibition via glibenclamide. More important, mitochondrial-derived reactive oxygen species sequester diminished AIM2-dependent IL-1 $\alpha$ release. Our data demonstrate that lung tumor-associated pDCs are responsive to the activation of AIM2 that promotes calcium efflux and reactive oxygen species from mitochondria, leading to calpain activation and high levels of IL-1 $\alpha$, which facilitate tumor cell proliferation in the lung. (Am J Pathol 2015, 185: 3115-3124; http://dx.doi.org/10.1016/j.ajpath.2015.07.009)
\end{abstract}

In the past decade, several studies have highlighted the key role of plasmacytoid dendritic cells (pDCs) in innate immunity. ${ }^{1,2}$ Their discovery dates back 50 years ago and, although their functional activity is nowadays being better explored, their role in specific pathologies still remains elusive. Human neoplasms, including solid tumors, such as head and neck, breast, ${ }^{3}$ ovarian, ${ }^{4}$ lung cancer, ${ }^{5}$ and skin tumors, ${ }^{6}$ are populated by nonactive/tolerogenic pDCs, associated with bad prognosis for cancer patients. ${ }^{7}$ Nonetheless, their function is still controversial. Various studies indicate that pDCs play an immunosuppressive role, and facilitate tumor progression in both animal models and humans. ${ }^{1}$ In contrast, others found that the presence of activated pDCs results in tumor regression in mice. ${ }^{1}$ Given these findings, it is clear that pDC function plays a critical role in tumor biology.
pDCs are recognized as type I interferon (IFN)-producing cells. ${ }^{8}$ The stimulation of $\mathrm{pDCs}$ with Toll-like receptor (TLR) 7 and 9 ligands leads to the production of large amounts of type I IFN, underlying their potential antiviral activity. 1,9 This function was also highlighted for tumor-associated pDCs (TApDCs), although pDC-derived type I IFN still needs clarification in that it can lead to both a mounting cytotoxic, antitumor activity ${ }^{10}$ and immunosuppression, ${ }^{11}$ which instead facilitates tumor cell immune escape. In a mouse model of melanoma, the intratumoral stimulation of pDCs with imiquimod, a TLR7 agonist, rendered these cells

Supported by FARB grant number FARB 2014 (University of Salerno), POR-FSE 2007-2013 Model Organism (MODO; R.S.), and POR-FSE 2007-2013 Research in Experimental Medicine (CREME; M.T. and M.P.).

Disclosures: None declared. 
cytotoxic and contributed to tumor regression via IFN receptor (IFNAR) signaling. ${ }^{12}$ Similarly, Liu et al $^{13}$ demonstrated that the intratumoral activation of $\mathrm{pDCs}$ via $\mathrm{CpG}$ could induce natural killer cell-dependent tumor regression. In sharp contrast, stimulation of lung tumor-bearing mice with systemic $\mathrm{CpG}$, a TLR9 ligand, did not lead to the same results. ${ }^{14,15} \mathrm{CpG}$-activated pDCs increased the recruitment of T-regulatory cells, and limited the inflammatory cell influx to the lung, establishing an immunosuppressive environment that favored tumor growth. ${ }^{1,15,16}$ The same was observed in a mouse model of breast cancer in which in vivo depletion of $\mathrm{pDCs}$ delayed tumor growth, showing that TApDCs provided an immune-subversive environment, most likely through $\mathrm{T}$ regulatory cell activation, thus favoring breast tumor progression. ${ }^{16}$ Similarly, the high presence of pDCs contrasted doxorubicin-induced tumor cell death and, thus, regression. ${ }^{17}$ In the absence of a specific stimulus, pDCs in the tumor mass have been associated with the development and maintenance of the immune-suppressive microenvironment. ${ }^{18}$ Similar to mice, human pDCs are in their immature phenotype in tumor masses. ${ }^{1,7}$ Nonetheless, a thorough study has never been conducted on the role of these cells in human lung tumor microenvironment, which may differ from the extensively studied mouse pDCs. The phenotype of pDCs is strictly correlated to the environment they encounter. ${ }^{1}$ In this context, chronic inflammation and the ensuing signaling pathways in the tumor mass are relevant to decide for the fate of the infiltrated pDCs.

An emerging area of investigation is the role of the inflammasome in cancer. ${ }^{19}$ The inflammasome is a multiprotein complex that comprises the assembly of Nod-like receptors (NLRs) or HIN200 family receptors, such as absent in melanoma 2 (AIM2), able to bind the adaptor apoptosis-associated speck-like protein containing a carboxyterminal CARD that induces the autocleavage of caspase- 1 and the activation of IL-1-like cytokines. ${ }^{19}$ It is of great interest that high serum concentrations of proinflammatory inflammasome-related cytokines (eg, IL- $1 \alpha / \beta$ and IL-18) are correlated to malignancies with a low rate of survival from time of diagnosis. ${ }^{20}$ In the tumor microenvironment, IL-1-like cytokines can be secreted by both malignant and infiltrated immune cells. ${ }^{21}$ On the basis of the notion that the inflammasome is responsible for IL-1like cytokine release by bone marrow-derived macrophages and dendritic cells, ${ }^{19}$ the aim of our study was to understand the role of pDCs during a pathological condition, such as lung cancer.

Herein, we found that human immunosuppressive TApDCs produced higher levels of IL- $1 \alpha$ than healthy pDCs in an AIM2-dependent manner and that their activity was type I IFN and oxidative stress dependent.

\section{Materials and Methods}

\section{Human Samples}

We used lung samples of non-small cell lung cancer patients undergoing thoracic surgery after their approval according to
Table 1 Histological Status of the Tumor Tissues Obtained by 14 Patients with Lung Cancer

\begin{tabular}{ll}
\hline Patient No. & Type of lung cancer \\
\hline 1 & Adenocarcinoma \\
2 & Adenocarcinoma \\
3 & Adenocarcinoma \\
4 & Squamous carcinoma \\
5 & Adenocarcinoma \\
6 & Adenocarcinoma \\
7 & Adenocarcinoma \\
8 & Squamous carcinoma \\
9 & Adenocarcinoma \\
10 & Adenocarcinoma \\
11 & Squamous carcinoma \\
12 & Adenocarcinoma \\
13 & Adenocarcinoma \\
14 & Squamous carcinoma \\
\hline
\end{tabular}

the Review Board of the Hospital of Salerno (Salerno, Italy). The non-small cell lung cancer subjects were $60 \pm 10$ years of age, and the histological status is described in Table 1. Samples from the tumor mass were defined as cancerous, whereas the healthy samples were obtained from the same patient from a distant noncancerous portion of the lung.

\section{Isolation of Human Lung-Derived pDCs}

pDCs were isolated from healthy and cancerous samples that were excised and digested with $1 \mathrm{U} / \mathrm{mL}$ collagenase and $20 \mu \mathrm{g} /$ $\mathrm{mL}$ DNase I (Sigma Aldrich, Milan, Italy) and antibiotics. Red blood cells were lysed. pDCs were isolated using a custom negative selection in that the enrichment immunomagnetic cocktail for human pDCs (EasySep Stem Cell, Voden, Milan, Italy) was modified with the addition of anti-EpCam antibody to avoid epithelial or tumor cell contamination. Purity was checked by flow cytometry, and was routinely approximately $85 \%$ to $90 \%$ (Supplemental Figure S1A), and by means of confocal microscopy (Supplemental Figure S1B). pDCs were treated with the following: 0.1 to $10 \mu \mathrm{g} / \mathrm{mL} \mathrm{CpG}$ (InvivoGen, San Diego, CA), 0.1 to $10 \mu \mathrm{g} / \mathrm{mL}$ imiquimod (InvivoGen), 0.1 to $10 \mu \mathrm{g} / \mathrm{mL}$ poly(dA:dT) (InvivoGen), $1 \mu \mathrm{mol} / \mathrm{L}$ glibenclamide, NLRP3 inhibitor (Sigma Aldrich, Rome, Italy), $1 \mu \mathrm{mol} /$ L diazoxide (DZX; Sigma Aldrich, Rome, Italy), $10 \mu \mathrm{mol} / \mathrm{L}$ calpain inhibitor MDL 28170 (Sigma Aldrich, Rome, Italy), $10 \mu \mathrm{mol} / \mathrm{L}$ necrostatin-1, receptor-interacting protein kinase inhibitor (Sigma Aldrich, Rome, Italy), $10 \mu \mathrm{mol} / \mathrm{L}$ diphenyleneiodonium chloride (DPI), NADPH oxidase inhibitor (Sigma Aldrich, Rome, Italy), $10 \mu \mathrm{mol} / \mathrm{L} N$-acetylcysteine (NAC; Sigma Aldrich, Rome, Italy), and $0.1 \mu \mathrm{g} / \mathrm{mL}$ LL-37 (Biolegend, San Diego, CA).

\section{Co-Culture Studies}

Carboxyfluorescein diacetate succinimyl ester $(\mathrm{CFSE})^{+}$ human leukemia K562 cells were cultured with pDCs (ratio, $1: 2.5)$ for 5 to 24 hours to measure their cytotoxic activity, according to the percentage of $\mathrm{CFSE}^{+}$propidium 

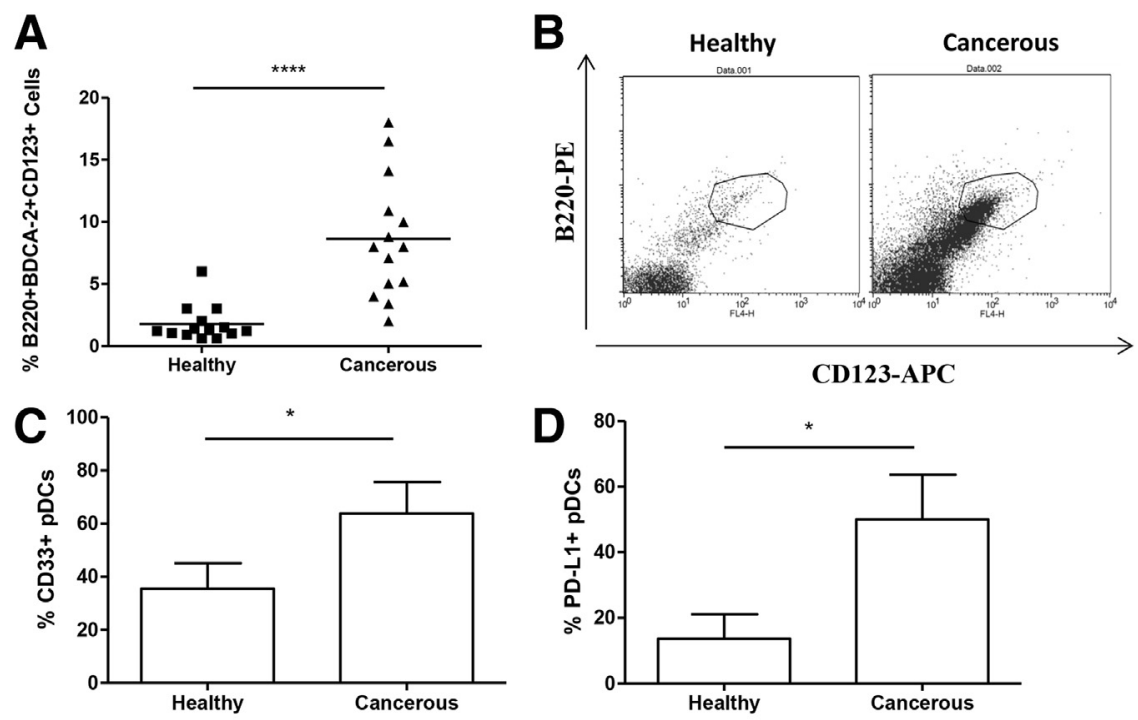

\begin{abstract}
Figure 1 Immunosuppressive plasmacytoid dendritic cells ( $p D C s)$ populate lung tumor mass. Human samples of non-small cell lung cancer (NSCLC) patients undergoing thoracic surgery were enzymatically digested, and $\mathrm{pDC}$ s were isolated by means of an immunomagnetic kit. A: Lung tumor masses (cancerous) present a higher percentage of pDCs than healthy lung tissues. B: $p D C s$ are identified as $B 220^{+} \mathrm{CD} 19^{-} \mathrm{BDCA}-2^{+} \mathrm{CD} 123^{+}$cells by means of flow cytometry. $\mathbf{C}-\mathbf{F}$ : The expression of CD33 (C), PD-L1 (D), HLA-AI (E), and HLA-D (F) is higher on cancerous tumor-associated pDCs than healthy cells. G: Intracellular staining of granzyme $B$ reveals higher presence of this cytotoxic mediator in cancerous than healthy PDCs. Data represent means \pm SEM $(\mathbf{C}-\mathbf{G}) . n=14$ patients. ${ }^{*} P<0.05,{ }^{* *} P<0.01,{ }^{* * *} P<0.005$, and $* * * * P<0.0001$, as determined by two-tailed unpaired $t$-test.
\end{abstract}
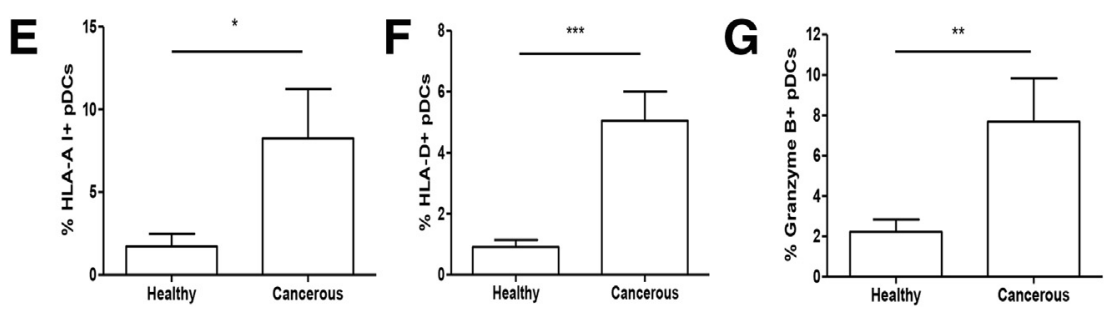

iodide-positive K562 cells determined by flow cytometry. In another set of experiments, we performed co-culture experiments with the human Jurkat $\mathrm{T}$ cell line and $\mathrm{CpG}$ or phosphate-buffered saline-pulsed pDCs (ratio, 1:10 or $1: 5)$, to test the activity of pDC antigen-presenting activity.

\section{Flow Cytometry Analysis}

pDCs were identified by flow cytometry (BD FacsCalibur, Milan, Italy) using the following antibodies: CD123allophycocyanin, B220-phycoerythrin, CD19-PeCy5.5, BDCA-2 fluorescein isothiocyanate (FITC), CD33-PerCP, HLA-AI-PerCP, HLA-D-PerCP, granzyme B-FITC or allophycocyanin, and PD-L1-FITC or allophycocyanin (eBioscience, San Diego, CA). Intracellular staining for IL- $1 \alpha$ was performed on fixed/permeabilized pDCs after the extracellular staining for CD123, B220, and CD19. Healthy and cancerous pDCs were stained for MitoSOX Mitochondrial Superoxide Indicator, as indicated in the manufacturer's guide (Life Technologies, Carlsbad, CA).

\section{Immunofluorescence Analysis}

Enriched pDC solution and human tissues were stained for CD123-AlexaFluor 555 (eBioscience) or BDCA-2AlexaFluor 555 (eBioscience) and intracellular IL-1 $\alpha-$ FITC. Similar staining was performed on human lung cancer-derived tissues. Images were observed by means of confocal microscopy (magnification, $\times 40$; Zeiss, Jena, Germany).

\section{Activity of Caspase-1}

Caspase- 1 activity was measured by means of a commercially available FAM FLICA caspase-1 assay kit (ImmunoChemistry Technologies, Bloomington, MN) and analyzed/expressed according to the absorbance $(550 \mathrm{~nm})$ of FLICA $^{+}$cells.

\section{Cytokine Measurements}

IFN $\alpha$, IL-18, IL- $1 \alpha$, and IL-1 $\beta$ were measured in cell-free supernatant obtained from the pDC culture by using commercially available enzyme-linked immunosorbent assays (eBioscience).

\section{Measurement of Intracellular Calcium}

Intracellular calcium concentrations were measured by using the fluorescent dye Fura 2-AM (Sigma Aldrich, Rome, Italy). pDCs $\left(5 \times 10^{4}\right.$ cells per well) were incubated at $37^{\circ} \mathrm{C}$ with $\mathrm{CpG}$, imiquimod, and poly(dA:dT) for 1 hour. Thereafter, cells were washed and Fura 2-AM hydrolysis was allowed in calcium-free medium, as already reported. ${ }^{22}$ Data were expressed as percentage of $\Delta$ increase of fluorescence ratio (F340/F380 $\mathrm{nm}$ ) induced by $1 \mu \mathrm{mol} / \mathrm{L}$ ionomycin or $0.05 \mu \mathrm{mol} / \mathrm{L}$ carbonyl cyanide p-trifluoromethoxy-phenylhydrazone-basal fluorescence/basal fluorescence ratio (F340/F380 nm).

\section{Statistical Analysis}

Results are expressed as means \pm SEM. A $t$-test or oneway analysis of variance was used to statistically 
A

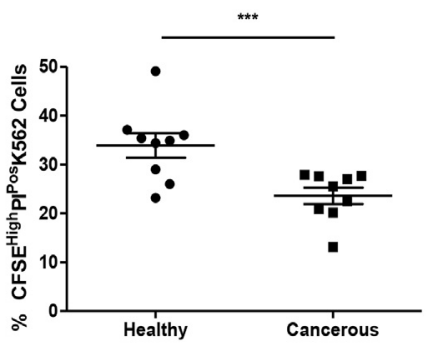

B

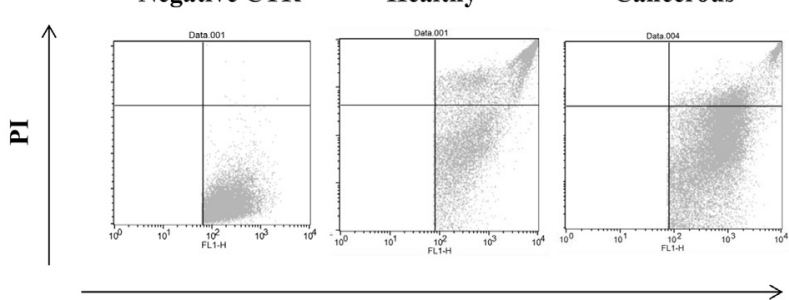

CFSE

Figure 2 Cancerous plasmacytoid dendritic cells ( $p D C s$ ) do not have cytotoxic activity. Co-culture experiments were performed between human pDCs and the leukemia cell line K562 cells (ratio, 2.5:1), previously stained with CFSE. Five hours after the addition of pDCs to K562 cells, flow cytometry analysis was performed by using propidium iodide (PI) to reveal $\mathrm{CFSE}^{+}$dying $\mathrm{K} 562$ cells. Cancerous pDCs do not induce K562 to cell death, contrary to healthy $\mathrm{pDCs}(\mathbf{A})$, as observed by the lower percentage of $\mathrm{CFSE}^{+} \mathrm{PI}^{+}$cells compared with $\mathrm{CFSE}^{+}$cells (B). Negative control (CTR) for flow cytometry analysis for CFSE ${ }^{+} \mathrm{K} 562$ cells without the presence of $\mathrm{pDCs}$ is shown. Data represent means $\pm \operatorname{SEM}(\mathbf{A}) . n=9$ patients. ${ }^{* *} P<0.005$, as determined by two-tailed unpaired $t$-test. CSFE, carboxyfluorescein diacetate succinimyl ester.

evaluate differences among treatments. $P<0.05$ was considered significant.

\section{Results}

\section{Human Lung Cancer Tissues Are Highly Populated by pDCs}

Previously, we demonstrated that pDCs highly populate the lung of tumor-bearing mice participating in the establishment of the tumor immune microenvironment and progression. ${ }^{14,15}$ To understand the role of pDCs in humans, samples of cancerous and healthy human lung were enzymatically digested and analyzed for the presence of pDCs (identified as B $220^{+} \mathrm{CD} 19^{-} \mathrm{BDCA}-2^{+} \mathrm{CD} 123^{+}$cells) by means of flow cytometry. Human lung cancer tissues were highly populated by pDCs compared with healthy lung tissues $(1.72 \pm 0.36$ versus $8.49 \pm 1.22$ ) (Figure $1, \mathrm{~A}$ and $\mathrm{B}$, and Supplemental Figure S1C), confirming our previous data on mice. ${ }^{15}$ Interestingly, we found that healthy lung tissues had similar percentages of pDCs as healthy blood (data not shown).

To understand the phenotype of pDCs, we went on by analyzing the expression of the immunosuppressive $\mathrm{CD} 33^{23}$ and PD-L1. ${ }^{24}$ Lung cancer-derived pDCs presented higher levels of CD33 (Figure 1C) and PD-L1 (Figure 1D), and they also had higher levels of HLA-AI (Figure 1E), HLA-D
(Figure 1F), and intracellular granzyme B (Figure 1G). Because of the high expression of HLAs, to evaluate pDC antigen-presenting activity, we performed co-culture experiments with the human Jurkat $\mathrm{T}$ cell line and $\mathrm{CpG}$ - or phosphate-buffered saline-pulsed pDCs (ratio, 1:10 or 1:5). The proliferation rate of Jurkat $T$ cells was increased when cancerous, but not healthy, pDCs were added. However, we were not able to detect type 1 helper T-cell-like cytokines to show an antitumor activity (data not shown).

According to the higher expression of granzyme $\mathrm{B}, \mathrm{pDCs}$ were co-cultured with the K562 cell line, previously stained with CFSE, to measure pDC cytotoxic activity. The addition of lung cancer-derived (cancerous) pDCs to $\mathrm{CFSE}^{+} \mathrm{K}_{562}$ cells significantly reduced the percentage of $\mathrm{CFSE}^{\text {high }}$ propidium iodide-positive cells (Figure 2A), but, instead, a higher percentage was registered for $\mathrm{CFSE}^{+}$propidium iodide-negative K562 cells (Figure 2B), compared with healthy pDCs, suggesting that K562 cells were induced to cell proliferation. These data highlight that cancerous immunosuppressive pDCs facilitate tumor cell proliferation in human lung cancer.

\section{Human Lung Cancer-Derived pDCs Produce IL-1-Like} Cytokines

pDCs are highly specialized at sensing nucleic acids via the intracellular pattern recognition receptors TLR7 and TLR9, which lead to the release of high levels of type I IFN ${ }^{1}$ Hence, pDCs are recognized as the professional IFN-producing cells. The stimulation of healthy pDCs with $0.1 \mu \mathrm{g} / \mathrm{mL} \mathrm{CpG}$ and 1 $\mu \mathrm{g} / \mathrm{mL}$ imiquimod significantly increased the production of IFN $\alpha$ (Figure 3, A and B). Instead, cancerous pDCs were not able to release higher levels of IFN $\alpha$ under $\mathrm{CpG}$ (Figure 3A) and imiquimod stimulation (Figure 3B), although the basal levels of IFN $\alpha$ in these cells were significantly higher than the basal levels of healthy pDCs (Figure 3, A and B). Because a recent study found that type I IFN negatively controls pDC turnover in that an overproduction of type I IFNs can lead to the death of pDCs during steady-state conditions and viral infections, ${ }^{8}$ we measured lactate dehydrogenase (LDH) release. Both healthy and cancerous pDCs were not dying cells because the levels of LDH were not as high as the positive control (Figure 3C).

On the basis of the notion that IL-1-like cytokines highly populate tumor masses,${ }^{20}$ we kept on our study by investigating whether cancerous pDCs were able to release IL- $1 \alpha$ and IL- $1 \beta$ under inflammasome activation. We first measured the expression of AIM2 and NLRP3 by means of flow cytometry. The levels of AIM2 (Figure 3, D and E) and NLRP3 (Figure 3, $\mathrm{E}$ and $\mathrm{F}$ ) were significantly increased in cancerous $\mathrm{pDCs}$ compared with healthy cells. Interestingly, the stimulation of healthy and cancerous pDCs with poly(dA:dT), an AIM2 ligand, did not increase the levels of IL- $1 \beta$ (Figure $4 \mathrm{~A}$ ), whereas the stimulation of TApDCs with a well-known NLRP3 activator, ATP, increased IL-1 $\beta$ levels compared with healthy pDCs (healthy pDCs: control versus ATP stimulated, 

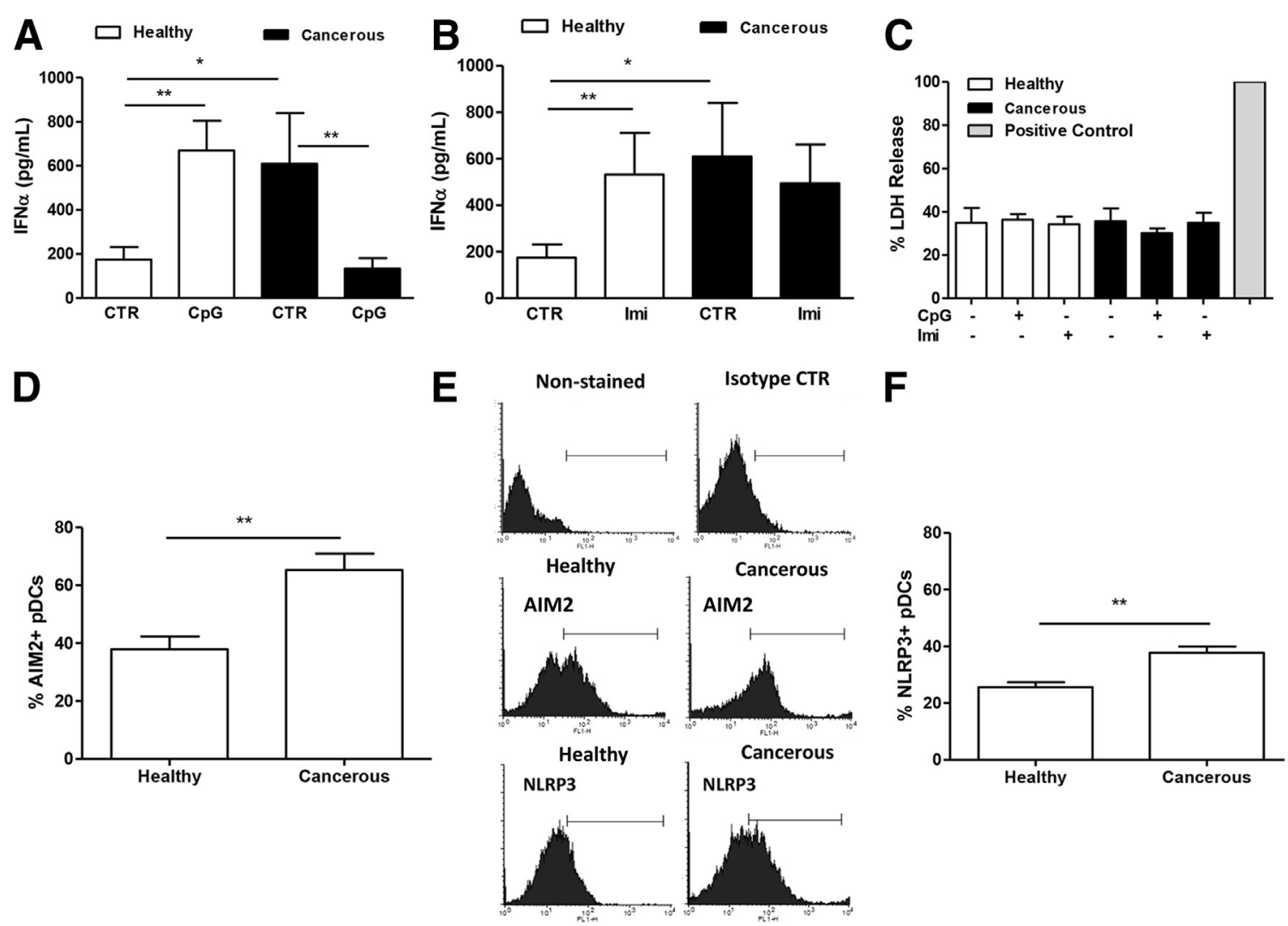

Figure 3 Cancerous plasmacytoid dendritic cells (pDCs) release higher levels of type I interferon (IFN). pDCs were stimulated with $0.1 \mu \mathrm{g} / \mathrm{mL} \mathrm{CpG}$ and 1 $\mu \mathrm{g} / \mathrm{mL}$ Imiquimod (Imi) for 5 hours. A and B: Healthy pDCs increase the production of IFN $\alpha$ after CpG (A) and Imi (B) stimulation. Instead, cancerous pDCs do not increase the release of IFN $\alpha$ under the same conditions, but have higher basal levels of IFN $\alpha$ compared with healthy pDCs. C: The release of lactate dehydrogenase (LDH), typical of dying cells, is not altered in both healthy and cancerous pDCs after CpG and Imi addition. D and F: Absent in melanoma 2 (AIM2; D) and Nod-like receptor P3 (NLRP3; F) expression was analyzed by means of flow cytometry. E: Representative data. Data represent means \pm SEM $(\mathbf{A}-\mathbf{D}$ and $\mathbf{F}) . n=14$ patients $(\mathbf{A}-\mathbf{D}$ and $\mathbf{F}) .{ }^{*} P<0.05,{ }^{*} * P<0.01$, as determined by two-tailed unpaired $t$-test. CTR, control.

$68.47 \pm 11.18$ versus $47.7 \pm 11.37$; TApDCs: control versus ATP stimulated, $75.88 \pm 12.66$ versus $142.9 \pm 13.3$; data not shown).

Interestingly, both healthy and cancerous pDCs showed higher levels of FAM-FLICA absorbance, confirming the activation of caspase-1 after poly(dA:dT) addition (Figure 4B), although these cells were not LDH-releasing cells (Figure 4C). In sharp contrast, poly(dA:dT) robustly increased the release of IL- $1 \alpha$ from cancerous pDCs compared with healthy pDCs (Figure 4D). To confirm that pDCs were the source of IL- $1 \alpha$ in our experimental conditions, we performed both confocal microscopy (Supplemental Figure S1, B and C) and flow cytometry analyses (Figure 4, E and F). Non-stimulated (control) lung cancer-derived pDCs $\left(\mathrm{CD} 123^{+}\right.$cells) had intracellular stores of IL-1 $\alpha$ (Supplemental Figure S1B). Concomitantly, the stimulation of lung cancer-derived pDCs with phosphate-buffered saline or poly(dA:dT) for 5 hours decreased the levels of the intracellular IL-1 $\alpha$ (Figure 4, E and $\mathrm{F}$ ), although not in a statistically significant manner $(P=0.0844)$, confirming the release of the cytokine into the supernatant, as shown in Figure 4D. Moreover, to avoid in vitro limitations, we also stained lung cancer tissues for intracellular IL-1 $\alpha$ (FITC). CD123 ${ }^{+}$cells were in the tumor microenvironment, surrounding the tumor mass, highlighted by the DAPI staining (Supplemental Figure S1C). CD123 ${ }^{+}$ cells were IL-1 $\alpha$-FITC positive, confirming that $\mathrm{pDCs}$ are another source of IL-1 $\alpha$ in the lung tumor microenvironment (Supplemental Figure S1C).

To further evaluate the role of the AIM2-caspase-1 inflammasome in cancerous pDCs, we treated the cells with $y$-Vad, a well-known caspase-1 inhibitor. The inhibition of caspase-1 significantly reduced the release of IL- $1 \alpha$ from cancerous pDCs after poly(dA:dT) stimulation (Figure 4G). The same effect was observed with an aspecific caspase inhibitor, z-VAD (Figure 4H).

To investigate the molecular mechanism underlying AIM2 activation, we went on by blocking type I IFNAR by means of a monoclonal antibody. The blockade of IFNAR reduced the release of IL- $1 \alpha$ after poly(dA:dT) treatment (Figure $4 \mathrm{I}$ ), implying that type I IFN is involved in the induction of IL- $1 \alpha$ after AIM2 activation. Moreover, to confirm the involvement of AIM2, we observed that the addition of LL-37, an AIM2 inhibitor, ${ }^{25}$ significantly reduced the levels of IL- $1 \alpha$ after poly(dA:dT) addition (Figure 4J). To confirm that poly(dA:dT) activity was solely AIM2 dependent, we treated TApDCs with a well-known inhibitor of NLRP $3,{ }^{26}$ glibenclamide, and a K/ATP channel opener, DZX. The inhibition of NLRP3 by means of glibenclamide did not alter 
A

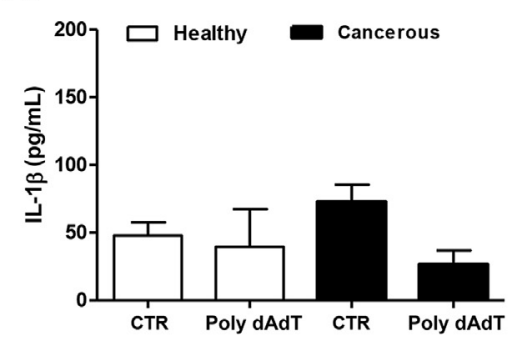

D

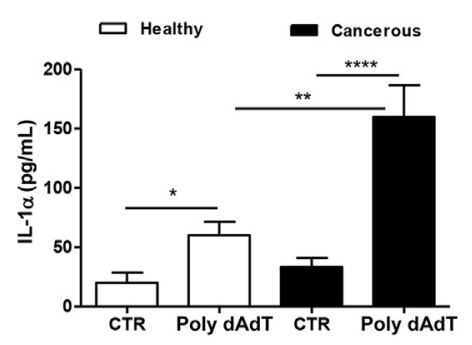

G

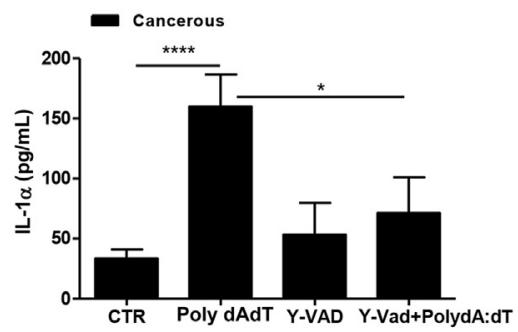

I

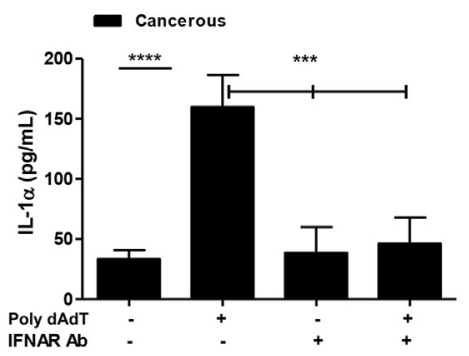

B

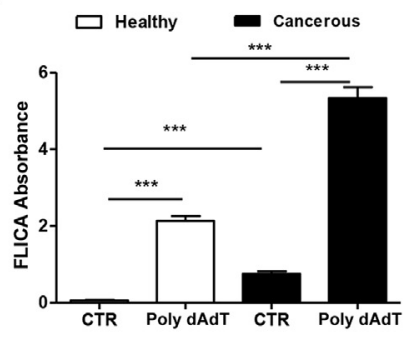

E

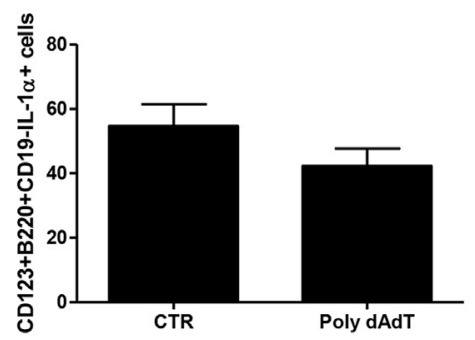

H

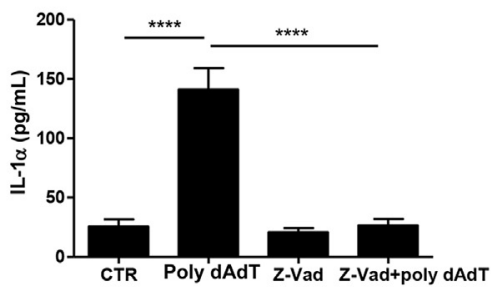

J

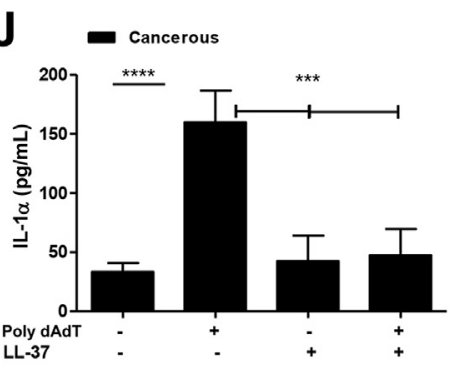

Figure 4 Plasmacytoid dendritic cells ( $\mathrm{pDCs}$ ) are responsive to absent in melanoma 2 (AIM2) activation. A-C: The activation of AIM2 by $1 \mu \mathrm{g} / \mathrm{mL}$ poly(dA:dT) does not increase the release of IL-1 $\beta$ (A) but instead significantly increases the levels of FAM-FLICA absorbance to confirm the activity of caspase-1 after the addition of poly(dA:dT) at 3 hours (B) onto human pDCs, which are not lactate dehydrogenase (LDH)-releasing cells ( 5 hours) (C). D and E: Poly(dA:dT) stimulation increases IL-1 $\alpha$ levels in cell-free supernatant (D) versus intracellular staining (E). F: Representative dot plots for flow cytometry analysis. G-J: The release of IL-1 $\alpha$ from cancerous $\mathrm{pDCs}$ is reduced by the inhibition of caspase-1 by $1 \mu \mathrm{mol} / \mathrm{L}$ y-VAD (specific; $\mathbf{G}), 1 \mu \mathrm{mol} / \mathrm{L}$ aspecific inhibitor z-VAD (H), the blockade of interferon receptor (IFNAR; I),

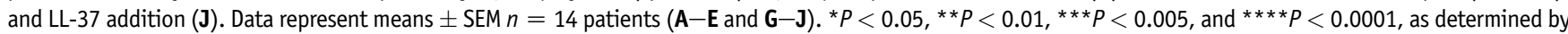
two-tailed unpaired $t$-test. Ab, antibody; APC, allophycocyanin; CTR, control; FITC, fluorescein isothiocyanate.

poly(dA:dT)-induced IL-1 $\alpha$ release (Figure 5A). Similarly, the activation of NLRP3 by means of DZX did not modify poly(dA:dT)-induced IL-1 $\alpha$ release (Figure 5B), implying that AIM2 was responsible for IL-1 $\alpha$ release.
Cell viability was not altered after the addition of glibenclamide and DZX (data not shown).

England et $\mathrm{al}^{27}$ described that IL- $1 \alpha$ is not a substrate for caspase-1. Similarly, Gross et $\mathrm{al}^{28}$ showed that $\mathrm{IL}-1 \alpha$ is not 


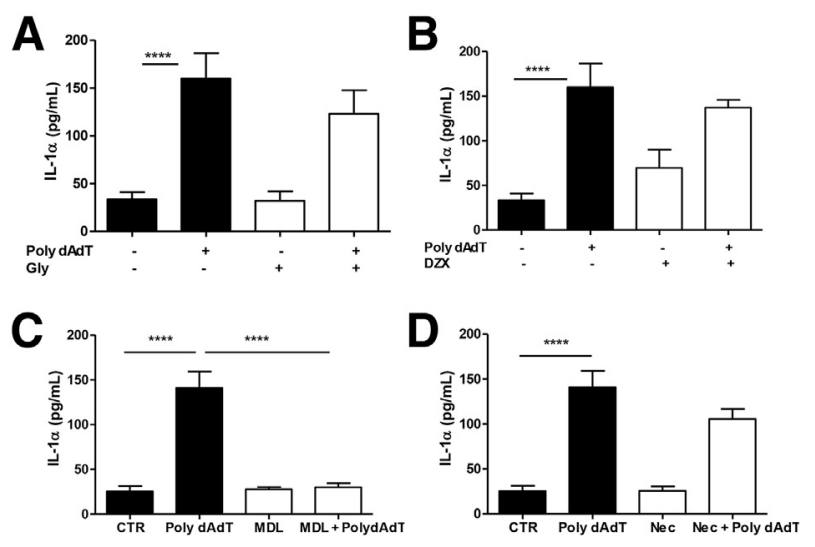

Figure 5 The release of IL- $1 \alpha$ after poly(dA:dT) administration is absent in melanoma 2 and calpain dependent. A: Tumor-associated plasmacytoid dendritic cells (TApDCs) show that the administration of $1 \mu \mathrm{mol} / \mathrm{L}$ Nod-like receptor P3 inhibitor, glibenclamide (Gly), does not alter IL-1 $\alpha$ release after poly(dA:dT) administration. B: The same is observed with 1 $\mu \mathrm{mol} / \mathrm{L}$ diazoxide (DZX). C and D: TApDCs were treated for 5 hours with 10 $\mu \mathrm{mol} / \mathrm{L}$ specific calpain inhibitor MDL 28170 (C) and $10 \mu \mathrm{mol} / \mathrm{L}$ receptorinteracting protein kinase $1 / 3$ inhibitor necrostatin-1 (Nec- 1 ; D). IL-1 $\alpha$ release was tested. Data represent means \pm SEM (A-D). $n=7$ patients (A-D). ${ }^{* * *} P<0.0001$, as determined by two-tailed unpaired $t$-test. CTR, control.

universally inflammasome dependent, but rather, calciumdependent calpain protease activity leads to IL- $1 \alpha$ processing. Therefore, to understand the cross talk between AIM2 inflammasome and calpain, we inhibited the calpain system by means of MDL 28170. Surprisingly, MDL robustly reduced IL-1 $\alpha$ release after poly(dA:dT) administration (Figure 5C), implying the involvement of calpain in an AIM2-dependent inflammasome. Moreover, to confirm that IL-1 $\alpha$ was not released under cell death, as already observed in Figure 4C, we treated TApDCs with necrostatin-1 to inhibit receptor-interacting protein kinase 1/3-dependent necrosis. The administration of necrostatin-1 did not alter $(P=0.0635)$ poly(dA:dT)-induced $\mathrm{IL}-1 \alpha$ release (Figure 5D). We did not observe any difference in LDH release after necrostatin-1 or MDL 28170 in the presence or absence of poly(dA:dT) (data not shown), implying that cell viability was not altered.

Taken together, these data suggest that poly(dA:dT) specifically triggers AIM2 inflammasome, which leads to caspase-1 but also calpain-dependent system activation, responsible for $\mathrm{IL}-1 \propto$ release in a type I IFN-dependent manner.

\section{Poly(dA:dT)-Mediated Release of IL-1-Like Cytokines Is Dependent on mtROS in Cancerous pDCs}

The activation of the inflammasome, especially in the case of NLRP3 inflammasome, has been widely related to mitochondrial-dependent oxidative stress. ${ }^{29}$ Given the notion that oxidative stress is highly registered in tumor masses and infiltrated immune cells, we administered inhibitors of reactive oxygen species (ROS) production. The release of IL- $1 \alpha$ after poly(dA:dT) from cancerous pDCs was significantly reduced by the addition of a classic ROS sequester, such as NAC (Figure 6A), and NADPH inhibitor, DPI (Figure 6B). We did not observe any difference in LDH release after NAC or DPI addition (data not shown).

Interestingly, the administration of poly(dA:dT) significantly increased the release of mitochondrial-derived ROS (mtROS), as observed by the positive staining for MitoSOX (Figure 6, C and E). Moreover, the specific inhibition of the mitochondrial NADPH by means of DPI significantly reduced MitoSOX ${ }^{+}$cells (Figure 6D) compared with NACand poly(dA:dT)-treated TApDCs. To confirm the role of mitochondria in the activation of AIM2 in cancerous pDCs, we measured the capability of pDCs to release mitochondrial calcium stores. The administration of poly $(\mathrm{dA}: \mathrm{dT})$ on healthy pDCs did not induce an increase in the release of total (Figure 6F) and mitochondrial (Figure 6G) calcium. Instead, both total (Figure 6F) and mitochondrial (Figure 6G) calcium stores were deprived of calcium that was released into the cytosol when poly(dA:dT) was added to cancerous pDCs. This effect was not reverted by the addition of glibenclamide, implying that NLRP3 is not involved in this signaling pathway (data not shown).

Taken together, these data imply that cancerous pDCs are responsive to the activation of AIM2 that promotes calcium efflux and ROS from mitochondria, leading to calpain activation that, in the end, leads to higher levels of IL- $1 \alpha$ from TApDCs. In their suppressive nature (Figures 1 and 2), the TApDCs favor tumor cell survival.

\section{Discussion}

In this study, we found that human lung cancer samples are highly populated by pDCs in their immunosuppressive phenotype, strictly correlated to the release of IL- $1 \alpha$ under AIM2 stimulation. Previously, we demonstrated that the immune-suppressive phenotype of lung tumor-associated pDCs was able to facilitate lung tumor growth in mice. ${ }^{14,15}$ This study, instead, further corroborates the protumor activity of pDCs in human lung cancer and focuses the attention on the inflammasome-derived IL-1-like cytokines correlated to the production of type I IFN.

The stimulation of AIM2 via poly(dA:dT) robustly augmented the release of IL- $1 \alpha$, but not of IL-1 $1 \beta$, from TApDCs in an IFN-, caspase-1-, and calpain-dependent manner.

AIM2 is a cytosolic double-stranded DNA sensor. ${ }^{30}$ Similarly to TLR7 and TLR9, AIM2 has been proposed to function as an immune surveillant for the invasion of viruses and bacteria as well as for the detection of endogenous danger-associated molecular patterns during autoimmune diseases. $^{25}$ However, neither IL-1 $\beta$ (Figure 4A) nor IL-18 and IL-33 (data not shown) were increased after AIM2 stimulation in TApDCs. Instead, we had higher levels of IL- $1 \alpha$ that did not correlate with the cytotoxic activity of TApDC, but, rather with the immunosuppressive nature of 

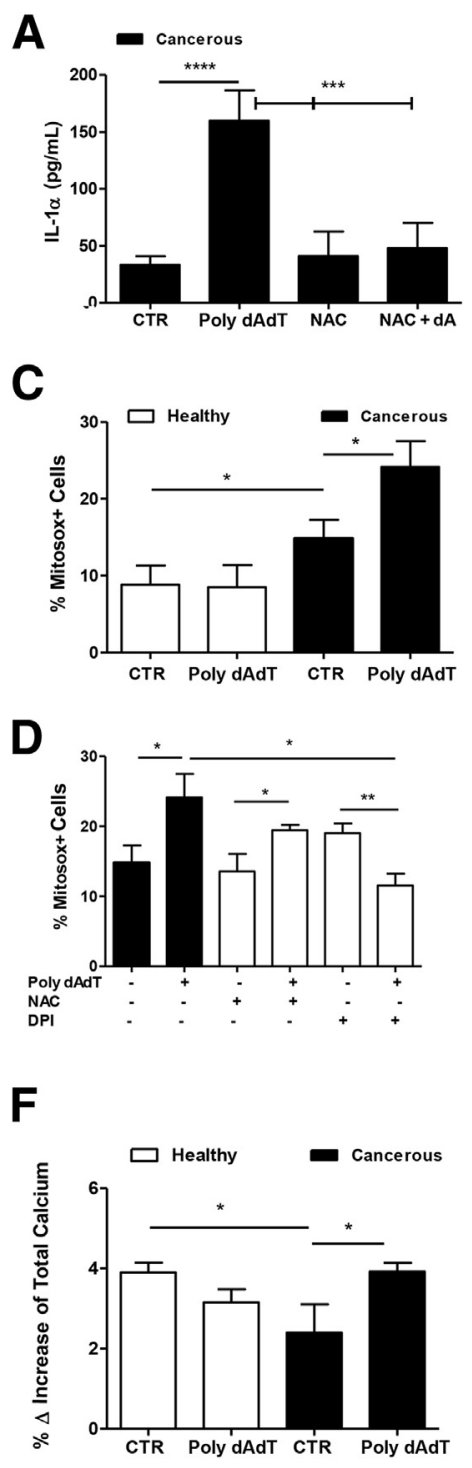

B

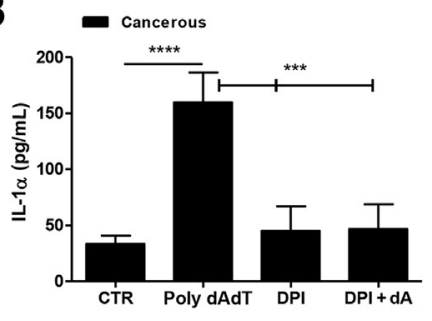

E
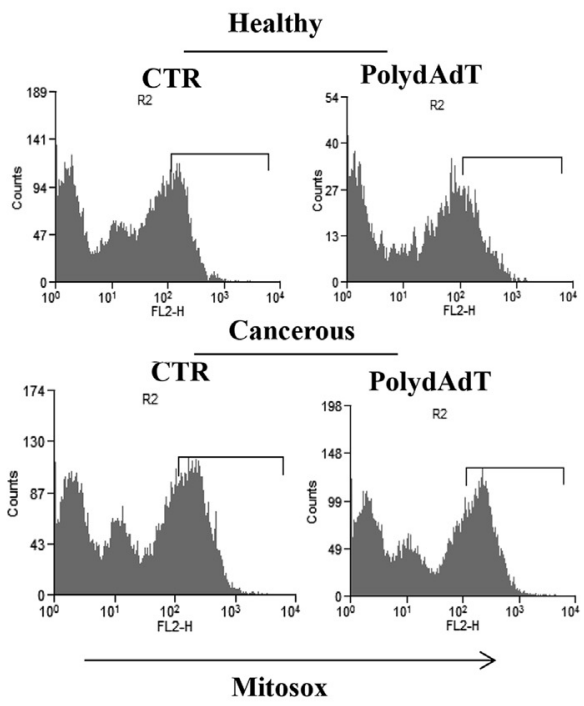

G

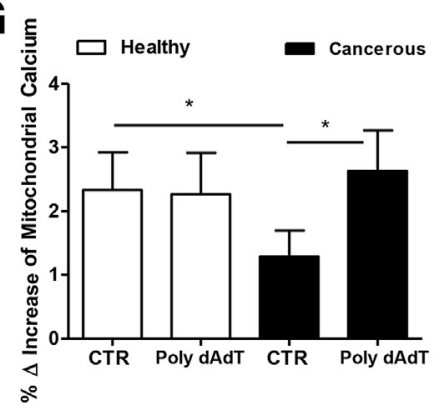

Figure 6 The activation of absent in melanoma 2 is dependent on the mitochondrial-derived oxidative stress. A and B: Poly(dA:dT)-induced IL- $1 \alpha$ release from cancerous plasmacytoid dendritic cells $(p D C s)$ is inhibited by the addition of $10 \mu \mathrm{mol} / \mathrm{L}$ $\mathrm{N}$-acetylcysteine (NAC; A) and $10 \mu \mathrm{mol} / \mathrm{L}$ diphenyleneiodonium chloride (DPI; B). Mitochondrialderived reactive oxygen species were measured by means of flow cytometry as MitoSOX-positive cells. C-E: Cancerous pDCs stimulated with poly(dA:dT) show higher positivity to MitoSOX than control (CTR) and healthy pDCs. Total (F) and mitochondrial (G) levels of calcium are significantly released into the cytosol of cancerous $\mathrm{pDCs}$ after poly $(d A: d T)$ stimulation. Data represent means $\pm \operatorname{SEM}(\mathbf{A}-\mathbf{D}, \mathbf{F}$ and $\mathbf{G}) . n=14$ patients $(\mathbf{A}-\mathbf{D}, \mathbf{F}$ and $\mathbf{G}) .{ }^{*} P<0.05,{ }^{*} P P<0.01$ $* * * P<0.005$, and $* * * * P<0.0001$, as determined by one-way analysis of variance and twotailed unpaired $t$-test. these cells. Instead, the activation of NLRP3 via ATP induced the release of both IL-1 $\beta$ and IL- $1 \alpha$ (data not shown).

ATP and double-stranded DNA derived from tumor dying cells can trigger NLRP3 and AIM2 inflammasome, respectively, although with a differential output in terms of IL-1-like cytokines. IL- $1 \alpha$ seems to be predominant in the lung tumor microenvironment, because tumor and epithelial cells are the major source of this cytokine. ${ }^{20}$

In this study, we demonstrated that TApDCs are another source of IL- $1 \alpha$ and can contribute to tumor proliferation. Indeed, IL- $1 \alpha$ is an alarmin that can facilitate tumor progression. In a model of diethylnitrosamine-induced liver carcinoma, skin papillomas, and gastric carcinoma, IL- $1 \alpha$ is released by dying cells, stimulating oxidative stress pathways responsible for local inflammation. In some cases, $\mathrm{IL}-1 \alpha$ is also produced during cell rescue from death to provide tissue regeneration and subsequent accumulation of mutations and tumor initiation and progression. ${ }^{19}$ Moreover, IL- $1 \alpha$ was described as antitumor in a mouse model of fibrosarcoma, ${ }^{31}$ because it promoted the cytotoxic natural killer, $\mathrm{CD} 4^{+}$, and $\mathrm{CD}^{+}{ }^{+} \mathrm{T}$ cells. Some cancer cells can express membrane IL-1 $\alpha$, which can increase the immunogenicity of tumor cells and promote antitumor immune surveillance and tumor regression. On the other side, however, high levels of released IL- $1 \alpha$ in the tumor microenvironment can favor angiogenesis and invasiveness ${ }^{31}$ and can be a marker for poor prognosis. ${ }^{20,21}$ Moreover, pDCs have been widely associated with the expansion of T-regulatory cells in cancer. ${ }^{1,16}$ However, some studies have shown that the adoptive transfer of pDCs pulsed with a tumor-associated antigen can exert antitumor activity in that it induces a cytotoxic adaptive immunity. The discrepancy between these evidences may underlie the nature of pDCs. We showed that the TApDC phenotype plays a pivotal role in cancer immunoediting in mice. ${ }^{14,15,17}$ Herein, we found that similar to mice, TApDCs have an immune-suppressive nature and are associated with 
lung tumor proliferation because of the activation of the AIM2-calpain axis that leads to IL- $1 \alpha$ release and activity.

The activation of AIM2 was reported as IFN-inducible inflammasome. ${ }^{25}$ Indeed, De Young et al ${ }^{32}$ suggested that AIM2 could be primed by TLR agonists that induce type I IFN release or by IFNs themselves. ${ }^{33,34}$ In our experimental conditions, we observed that the basal levels of IFN $\alpha$ from TApDCs were significantly higher than healthy. To confirm the role of type I IFN, the blockade of IFNAR significantly reduced AIM2-dependent IL- $1 \alpha$ release from TApDCs.

Similarly, Fang et $\mathrm{al}^{33}$ found that Streptococcus pneumoniae-infected macrophages required type I IFN signaling for the activation of AIM2 inflammasome. Gross

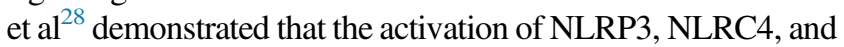
AIM2 inflammasomes can lead to the release of IL- $1 \alpha$, but not in a direct manner. In this scenario, the authors showed that IL$1 \alpha$ was not universally inflammasome dependent, but rather had calcium-dependent calpain protease activity. A support to this article is that IL- $1 \alpha$ is not a substrate for caspase-1, although some danger molecules can regulate inflammasome-dependent processing and release of $\mathrm{IL}-1 \alpha$ in mouse bone marrowderived macrophages. In this context, we found that calpain is involved in AIM2-dependent IL- $1 \alpha$ release in TApDCs. However, because of the nature of TApDCs, we were not able to knock down caspase-1 to discriminate its role compared with calpain. Nevertheless, we found that the activation of AIM2 via poly(dA:dT) led to mitochondrial release of calcium, which is important for calpain activation. Therefore, we could speculate that AIM2-induced mitochondrial calcium release is responsible for calpain activation that turns out with higher levels of IL- $1 \alpha$ from TApDCs. However, we found that $y$-VAd administration reduced the release of IL- $1 \alpha$ under poly(dA:dT) stimulation, most probably implying that this inhibitor is not as specific as reported, but most probably interferes with other caspase activity.

As reported by Gross et al, ${ }^{28}$ AIM2 activation leads to an increase in mtROS production in a caspase-1-dependent manner. Similarly, we found that poly(dA:dT) increased the release of calcium from mitochondrial stores, a connection point between the inflammasome and calpain activation. In support, the administration of DZX, an inducer of NLRP3, did not alter IL- $1 \alpha$ release after poly(dA:dT), implying, rather than an aspecific activity of poly(dA:dT) (Figure 5A), an involvement of calcium stores. Indeed, DZX is a K/ATP-channel opener and its activity increased (data not shown) the release of calcium from mitochondrial stores, further confirming the role of calcium in poly(dA:dT) activity in TApDCs. Poly(dA:dT) significantly induced IL- $1 \alpha$ after the activation of the mitochondrial-derived oxidative stress signaling, as observed by a higher percentage of MitoSOXpositive cells. It was recently reported that cell distress subsequent to mitochondrial dysfunction and the oxidation of many cell targets, including mitochondrial DNA, is able to induce IL-1-like cytokine release. ${ }^{29}$

Our study is the first to define a role for mtROS under AIM2 activation in tumor-derived pDCs. Although mtROS were described as NLRP3 stimuli, herein we demonstrate that cancerous pDCs are more susceptible to mitochondria dysfunction in that AIM2 leads to IL- $1 \alpha$ release. The difference between our study and the others' is that the cells were not undergoing cell death, and we principally detected significant increases in IL- $1 \alpha$ rather than IL- $1 \beta$. These effects may underlie the difference between mouse and human monocytes, dendritic cells, and macrophages. All studies conducted so far have been performed on mouse cultured bone marrow-derived cells rather than on pathological human disease-derived DCs.

Therefore, as already stated by Gross et $\mathrm{al}^{28}$ and as observed by our data, it is plausible to conclude that, although the events downstream of inflammasome signaling and calcium influx are in principle independent to one another, they can regulate common processes for IL- $1 \alpha$ release, an immunosuppressive cytokine responsible for the progression of lung malignancy.

Several mechanisms have been postulated for the immunosuppressive nature of $\mathrm{TApDCs}^{1}:$ i) release of tolerogenic factors, ii) immunoglobulin-like transcript 7 expression, iii) programed death-ligand 1 (PD-L1) expression, iv) Siglec family activity, and v) induction of a type 2 helper T-cell-like environment. Herein, we highlighted a novel mechanism by which pDCs can favor tumor cell survival. The activation of the AIM2 inflammasome can lead to mtROS and calcium influx, which lead to calpain activation, a mechanism that underlies the TApDC phenotype that favors tumor growth via IL- $1 \alpha$.

In conclusion, our data demonstrate that the release of IL- $1 \alpha$ via the activation of AIM2 by the autocrine activity of type I IFN or by a still unknown endogenous tumor-associated danger-associated molecular pattern that leads to the release of mtROS plays a crucial role for the immunosuppressive activity of TApDCs. Therefore, strategies aiming at modulating AIM2 activity in TApDC in the tumor site might prove to limit tumor cell proliferation.

\section{Supplemental Data}

Supplemental material for this article can be found at http://dx.doi.org/10.1016/j.ajpath.2015.07.009.

\section{References}

1. Pinto A, Rega A, Crother TR, Sorrentino R: Plasmacytoid dendritic cells and their therapeutic activity in cancer. Oncoimmunology 2012, 1:726-734

2. Vermi W, Soncini M, Melocchi L, Sozzani S, Facchetti F: Plasmacytoid dendritic cells and cancer. J Leukoc Biol 2011, 90: 681-690

3. Treilleux I, Blay J-Y, Bendriss-Vermare N, Ray-Coquard I, Bachelot T, Guastalla J-P, Bremond A, Goddard S, Pin JJ, BarthelemyDubois C, Lebecque S: Dendritic cell infiltration and prognosis of early stage breast cancer. Clin Cancer Res 2004, 10:7466-7474

4. Conrad C, Gregorio J, Wang Y-H, Ito T, Meller S, Hanabuchi S, Anderson S, Atkinson N, Ramirez PT, Liu YJ, Freedman R, Gilliet M: Plasmacytoid dendritic cells promote immunosuppression in ovarian cancer via ICOS costimulation of Foxp3(+) T-regulatory cells. Cancer Res 2012, 72:5240-5249 
5. Pinto A, Morello S, Sorrentino R: Lung cancer and Toll-like receptors. Cancer Immunol Immunother 2011, 60:1211-1220

6. Gerlini G, Urso C, Mariotti G, Di Gennaro P, Palli D, Brandani P, Salvadori A, Pimpinelli N, Reali UM, Borgognoni L: Plasmacytoid dendritic cells represent a major dendritic cell subset in sentinel lymph nodes of melanoma patients and accumulate in metastatic nodes. Clin Immunol 2007, 125:184-193

7. Chaput N, Conforti R, Viaud S, Spatz A, Zitvogel L: The Janus face of dendritic cells in cancer. Oncogene 2008, 27:5920-5931

8. Swiecki M, Wang Y, Vermi W, Gilfillan S, Schreiber RD, Colonna M: Type I interferon negatively controls plasmacytoid dendritic cell numbers in vivo. J Exp Med 2011, 208:2367-2374

9. McKenna K, Beignon A-S, Bhardwaj N: Plasmacytoid dendritic cells: linking innate and adaptive immunity. J Virol 2005, 79:17-27

10. Swann JB, Hayakawa Y, Zerafa N, Sheehan KCF, Scott B, Schreiber RD, Hertzog P, Smyth MJ: Type I IFN contributes to NK cell homeostasis, activation, and antitumor function. J Immunol 2007, 178:7540-7549

11. Gough DJ, Messina NL, Clarke CJP, Johnstone RW, Levy DE: Constitutive type I interferon modulates homeostatic balance through tonic signaling. Immunity 2012, 36:166-174

12. Drobits B, Holcmann M, Amberg N, Swiecki M, Grundtner R, Hammer M, Colonna M, Sibilia M: Imiquimod clears tumors in mice independent of adaptive immunity by converting pDCs into tumorkilling effector cells. J Clin Invest 2012, 122:575-585

13. Liu C, Lou Y, Lizée G, Qin H, Liu S, Rabinovich B, Kim GJ, Wang YH, Ye Y, Sikora AG, Overwijk WW, Liu YJ, Wang G, Hwu P: Plasmacytoid dendritic cells induce NK cell-dependent, tumor antigenspecific $\mathrm{T}$ cell cross-priming and tumor regression in mice. J Clin Invest 2008, 118:1165-1175

14. Rega A, Terlizzi M, Luciano A, Forte G, Crother TR, Arra C, Arditi M, Pinto A, Sorrentino R: Plasmacytoid dendritic cells play a key role in tumor progression in lipopolysaccharide-stimulated lung tumor-bearing mice. J Immunol 2013, 190:2391-2402

15. Sorrentino R, Morello S, Luciano A, Crother TR, Maiolino P, Bonavita E, Arra C, Adcock IM, Arditi M, Pinto A: Plasmacytoid dendritic cells alter the antitumor activity of CpG-oligodeoxynucleotides in a mouse model of lung carcinoma. J Immunol 2010, 185:4641-4650

16. Sisirak V, Faget J, Gobert M, Goutagny N, Vey N, Treilleux I, Renaudineau S, Poyet G, Labidi-Galy SI, Goddard-Leon S, Durand I, Le Mercier I, Bajard A, Bachelot T, Puisieux A, Puisieux I, Blay JY, Ménétrier-Caux C, Caux C, Bendriss-Vermare N: Impaired IFN- $\alpha$ production by plasmacytoid dendritic cells favors regulatory T-cell expansion that may contribute to breast cancer progression. Cancer Res 2012, 72:5188-5197

17. Terlizzi M, Popolo A, Pinto A, Sorrentino R: Plasmacytoid dendritic cells contribute to doxorubicin-induced tumor arrest in a mouse model of pulmonary metastasis. J Immunother 2014, 37:214-224

18. Lande R, Gilliet M: Plasmacytoid dendritic cells: key players in the initiation and regulation of immune responses. Ann N Y Acad Sci 2010, 1183:89-103

19. Terlizzi M, Casolaro V, Pinto A, Sorrentino R: Inflammasome: cancer's friend or foe? Pharmacol Ther 2014, 143:24-33
20. Dinarello CA: Interleukin- $1 \alpha$ neutralisation in patients with cancer. Lancet Oncol 2014, 15:552-553

21. Garlanda C, Dinarello CA, Mantovani A: The interleukin-1 family: back to the future. Immunity 2013, 39:1003-1018

22. Pecoraro M, Sorrentino R, Franceschelli S, Del Pizzo M, Pinto A, Popolo A: Doxorubicin-mediated cardiotoxicity: role of mitochondrial connexin 43. Cardiovasc Toxicol 2015, 15:366-376

23. Pillai S, Netravali IA, Cariappa A, Mattoo H: Siglecs and immune regulation. Annu Rev Immunol 2012, 30:357-392

24. Francisco LM, Sage PT, Sharpe AH: The PD-1 pathway in tolerance and autoimmunity. Immunol Rev 2010, 236:219-242

25. Rathinam VAK, Jiang Z, Waggoner SN, Sharma S, Cole LE, Waggoner L, Vanaja SK, Monks BG, Ganesan S, Latz E, Hornung V, Vogel SN, Szomolanyi-Tsuda E, Fitzgerald KA: The AIM2 inflammasome is essential for host defense against cytosolic bacteria and DNA viruses. Nat Immunol 2010, 11:395-402

26. Lamkanfi M, Mueller JL, Vitari AC, Misaghi S, Fedorova A, Deshayes K, Lee WP, Hoffman HM, Dixit VM: Glyburide inhibits the Cryopyrin/Nalp3 inflammasome. J Cell Biol 2009, 187:61-70

27. England H, Summersgill HR, Edye ME, Rothwell NJ, Brough D: Release of interleukin- $1 \alpha$ or interleukin- $1 \beta$ depends on mechanism of cell death. J Biol Chem 2014, 289:15942-15950

28. Gross O, Yazdi AS, Thomas CJ, Masin M, Heinz LX, Guarda G, Quadroni M, Drexler SK, Tschopp J: Inflammasome activators induce interleukin- $1 \alpha$ secretion via distinct pathways with differential requirement for the protease function of caspase-1. Immunity 2012, 36: $388-400$

29. Shimada K, Crother TR, Karlin J, Dagvadorj J, Chiba N, Chen S, Ramanujan VK, Wolf AJ, Vergnes L, Ojcius DM, Rentsendorj A, Vargas M, Guerrero C, Wang Y, Fitzgerald KA, Underhill DM, Town T, Arditi M: Oxidized mitochondrial DNA activates the NLRP3 inflammasome during apoptosis. Immunity 2012, 36 : 401-414

30. Jin T, Perry A, Jiang J, Smith P, Curry JA, Unterholzner L, Jiang Z, Horvath G, Rathinam VA, Johnstone RW, Hornung V, Latz E, Bowie AG, Fitzgerald KA, Xiao TS: Structures of the HIN domain: DNA complexes reveal ligand binding and activation mechanisms of the AIM2 inflammasome and IFI16 receptor. Immunity 2012, 36 : $561-571$

31. Rider P, Carmi Y, Voronov E, Apte RN: Interleukin-1 $\alpha$. Semin Immunol 2013, 25:430-438

32. DeYoung KL, Ray ME, Su YA, Anzick SL, Johnstone RW, Trapani JA, Meltzer PS, Trent JM: Cloning a novel member of the human interferon-inducible gene family associated with control of tumorigenicity in a model of human melanoma. Oncogene 1997, 15: $453-457$

33. Fang R, Hara H, Sakai S, Hernandez-Cuellar E, Mitsuyama M, Kawamura I, Tsuchiya K: Type I interferon signaling regulates activation of the absent in melanoma 2 inflammasome during Streptococcus pneumoniae infection. Infect Immun 2014, 82:2310-2317

34. Belhocine K, Monack DM: Francisella infection triggers activation of the AIM2 inflammasome in murine dendritic cells. Cell Microbiol 2012, 14:71-80 